\title{
Effect of Ion Composition on Oblique Magnetosonic Waves*)
}

\author{
Yuichi KONDO and Mieko TOIDA \\ Department of Physics, Nagoya University, Nagoya 464-8602, Japan \\ (Received 6 December 2010 / Accepted 13 January 2011)
}

\begin{abstract}
The effects of ion composition on oblique magnetosonic waves in a two-ion-species plasma are studied theoretically and numerically. First, it is analytically shown that the KdV equation for the low-frequency mode, the lower branch of magnetosonic waves, is valid for amplitudes $\varepsilon<\varepsilon_{\max }^{(1-)}$, where $\varepsilon_{\max }^{(1-)}$ is a measure of the upper limit of the amplitude of the rarefactive solitary pulse of the low-frequency mode and is given as a function of the propagation angle of the wave $\theta$, the density ratio and cyclotron frequency ratio of two ion species. The value of $\varepsilon_{\max }^{(1-)}$ increases with decreasing $\theta$. Next, with electromagnetic particle simulations, the nonlinear evolution of the low- and high-frequency modes is examined. It is demonstrated that shorter-wavelength low- and high-frequencymode waves are generated from a long-wavelength low-frequency-mode pulse if its amplitude $\varepsilon$ exceeds $\varepsilon_{\max }^{(1-)}$.
\end{abstract}

(c) 2011 The Japan Society of Plasma Science and Nuclear Fusion Research

Keywords: magnetosonic wave, nonlinear wave, soliton, $\mathrm{KdV}$ equation, multi-ion-species plasma

DOI: $10.1585 /$ pfr.6.2401026

\section{Introduction}

Nonlinear magnetosonic waves have been investigated by many authors since the waves play crucial roles in particle heating and acceleration in space and fusion plasmas. Furthermore, because both plasmas usually contain multiple ion species, the effects of multiple ion species on the magnetosonic waves have been extensively studied.

In a two-ion-species plasma, the magnetosonic wave propagating perpendicular to a magnetic field is split into two modes; the low- and high-frequency modes. The frequencies of the low-frequency mode are in the range $0<\omega<\omega_{-r}$, where $\omega_{-r}$ is the ion-ion hybrid resonance frequency [1],

$$
\omega_{-\mathrm{r}}=\left[\left(\omega_{\mathrm{p} a}^{2} \Omega_{b}^{2}+\omega_{\mathrm{p} b}^{2} \Omega_{a}^{2}\right) /\left(\omega_{\mathrm{p} a}^{2}+\omega_{\mathrm{p} b}^{2}\right)\right]^{1 / 2} .
$$

Here, the subscripts $a$ and $b$ indicate ion species, and $\Omega_{j}$ and $\omega_{\mathrm{p} j}(j=a$ or $b)$ represent their cyclotron and plasma frequencies, respectively. The high-frequency mode has a finite cut-off frequency given by

$$
\omega_{+0}=\left(\omega_{\mathrm{p} a}^{2} / \Omega_{a}^{2}+\omega_{\mathrm{p} b}^{2} / \Omega_{b}^{2}\right) \Omega_{a} \Omega_{b}\left|\Omega_{\mathrm{e}}\right| / \omega_{\mathrm{pe}}^{2} .
$$

Here, the subscript e refers to the electrons.

Although the dispersion curves of the high- and lowfrequency modes are quite different in the long-wavelength region, Korteweg-de Vries ( $\mathrm{KdV}$ ) equations have been derived for both the low- and high-frequency modes [2]. The characteristic soliton width of the low-frequency mode is of the order of the ion inertia length $c / \omega_{\mathrm{pi}}$, while that of the high-frequency mode is of the order of the electron skin depth $c / \omega_{\text {pe }}$. The KdV equation for the high-frequency mode is valid for wave amplitudes $\left(m_{\mathrm{e}} / m_{\mathrm{i}}\right)^{1 / 2} \ll \varepsilon \ll 1$.

author'se-mail: toida@cc.nagoya-u.ac.jp

*) This article is based on the presentation at the 20th International Toki Conference (ITC20).
The nonlinear evolution of these modes have been studied in Ref. [3], under the assumption that the density of the hydrogen $(\mathrm{H})$ ions is much higher than heavy-ion densities as in space plasmas. In such plasma, if a large-amplitude, long-wavelength pulse of the low-frequency mode is excited, it will steepen and then emit short-wavelength pulses of the high-frequency mode.

Fusion plasmas also contain multiple ion species, such as deuterium (D) and tritium (T). However, the densities of $\mathrm{D}$ and $\mathrm{T}$ are the same order, which differs from the case of space plasmas. In view of these facts, the reference [4] has discussed the effect of ion mass and density ratios on perpendicular magnetosonic waves. It has pointed out the importance of the normalized frequency difference,

$$
\Delta_{\omega}=\left(\omega_{+0}-\omega_{-\mathrm{r}}\right) / \omega_{+0},
$$

in the nonlinear evolution of the waves. The value of $\Delta_{\omega}$ increases with increasing $\Omega_{a} / \Omega_{b}$, where $\Omega_{a}>\Omega_{b}$ is assumed. For a fixed $\Omega_{a} / \Omega_{b}, \Delta_{\omega}$ has its maximum value when the ion charge densities are equal, $n_{a} q_{a}=n_{b} q_{b}$. It has been found that the $\mathrm{KdV}$ equation for the low-frequency mode is valid for the amplitudes $\varepsilon<2 \Delta_{\omega}$, which suggest that nonlinear coupling between the high- and low-frequency modes can occur if $\varepsilon>2 \Delta_{\omega}$. With electromagnetic particle simulations, nonlinear evolution of magnetosonic waves was investigated for various values of $n_{a} / n_{b}$ and $\Omega_{a} / \Omega_{b}$, and has been shown that high-frequency-mode pulses are generated from a long-wavelength low-frequency-mode pulse if its amplitude exceeds $2 \Delta_{\omega}$.

In this paper, we extend the above work to the study of oblique magnetosonic waves. In Sec. 2, we present theoretical expressions for quantities on the low-frequency mode, including $\Delta_{\omega}$ as a factor. We then obtain the condition for the $\mathrm{KdV}$ equation for the low-frequency mode to be 
valid. In Sec. 3, we investigate, with electromagnetic particle simulations, how the nonlinear evolution of the oblique magnetosonic waves depends on $\Delta_{\omega}$ and the propagation angle $\theta$.

\section{Theory for Low-Frequency Mode}

We briefly outline the linear dispersion relations of magnetosonic waves in a multi-ion-species plasma. We consider the waves propagating in the $x$ direction in a magnetic field in a $(x, z)$ plane, $\boldsymbol{B}_{0}=B_{0}(\cos \theta, 0, \sin \theta)$. Figure 1 shows dispersion curves of magnetohydrodynamic waves for propagation angle $\theta=86^{\circ}$ in a $\mathrm{H}-\mathrm{He}$ plasma with $n_{\mathrm{H}} / n_{\mathrm{He}}=10$. The lines $\mathrm{A}, \mathrm{H}$ and $\mathrm{L}$ represent the Alfven wave, the high- and low-frequency modes of the magnetosonic wave, respectively; the properties of the Alfven wave is not significantly changed by the presence of two ion species. The dispersion curves for both the high- and low-frequency modes have large curvature at the wavenumber $k_{\mathrm{c}}$ defined by

$$
k_{\mathrm{c}}=\omega_{-\mathrm{r}} / v_{\mathrm{A}},
$$

where $v_{\mathrm{A}}$ is the Alfven speed. The two curves are close near this wavenumber.

We now investigate the linear dispersion relation of the low-frequency mode in detail. For the range of wavenumbers, $k \ll k_{\mathrm{c}}$, we can approximate the linear dispersion relation of the low-frequency mode as

$$
\frac{\omega^{2}}{v_{A}^{2} k^{2}} \simeq 1-\frac{2 \Delta_{\omega}}{\sin ^{2} \theta} \frac{k^{2}}{k_{\mathrm{c}}^{2}}+\frac{r}{\tan ^{2} \theta} \frac{k^{2}}{k_{\mathrm{c}}^{2}}-\frac{2 \Delta_{\omega}}{\sin ^{4} \theta} \frac{k^{4}}{k_{\mathrm{c}}^{4}},
$$

where $r$ is defined as

$$
r=\omega_{-\mathrm{r}}^{2}\left(\Omega_{a}^{2}+\Omega_{b}^{2}\right) /\left(\Omega_{a}^{2} \Omega_{b}^{2}\right)-1 .
$$

We introduce the angle $\theta_{\mathrm{cl}}$ defined by

$$
\cos ^{2} \theta_{\mathrm{cl}}=2 \Delta_{\omega} / r
$$

At the angle $\theta_{\mathrm{cl}}$, the second and third terms on the righthand side of Eq. (5) cancel. In the following, we consider the cases where $\theta<\theta_{\mathrm{cl}}$.

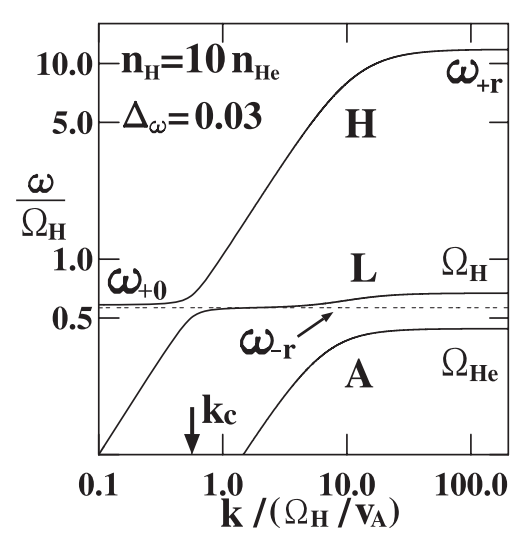

Fig. 1 Dispersion curves for magnetohydorodynamic waves for $\theta=86^{\circ}$ in a $\mathrm{H}-\mathrm{He}$ plasma with $n_{\mathrm{H}}=10 n_{\mathrm{He}}$.
When $\theta<\theta_{\mathrm{cl}}$, the dispersion curve of the lowfrequency mode has an inflection point in the region, $0<$ $k<k_{\mathrm{c}}$. For $k<k_{\text {inf }}^{(\mathrm{l})}$, where $k_{\mathrm{inf}}^{(\mathrm{l})}$ denotes the wave number of the inflection point, $\partial^{2} \omega / \partial^{2} k$ is positive, while for $k>k_{\text {inf }}^{(\mathrm{l})}, \partial^{2} \omega / \partial^{2} k$ is negative. One can obtain $k_{\text {inf }}^{(\mathrm{l})}$ from Eq. (5), applying the condition $\partial^{2} \omega / \partial^{2} k=0$, as

$$
\frac{k_{\mathrm{inf}}^{(1)}}{k_{\mathrm{c}}}=\left[\frac{3 \sin ^{2} \theta\left(r \cos ^{2} \theta-2 \Delta_{\omega}\right)}{20 \Delta_{\omega}-2\left(r \cos ^{2} \theta-2 \Delta_{\omega}\right)^{2}}\right]^{1 / 2} .
$$

For small wave numbers

$$
k \ll k_{\text {inf }}^{(1)}
$$

the dispersion relation (5) is written as

$$
\omega=v_{\mathrm{A}} k\left(1+d_{11}^{2} k^{2} / 2\right)
$$

Here, $d_{11}$ is defined as

$$
d_{11}=\left|2 \Delta_{\omega}-r \cos ^{2} \theta\right|^{1 / 2} /\left(k_{\mathrm{c}} \sin \theta\right) .
$$

As expected from Eq. (10), the nonlinear behavior of the low-frequency mode can be described by KdV equation $[5]$,

$$
\frac{\partial B_{z 1}}{\partial \tau}+\frac{3 v_{\mathrm{A}}}{2 B_{0}} \sin \theta B_{z 1} \frac{\partial B_{z 1}}{\partial \xi}-\frac{v_{\mathrm{A}} d_{l 1}^{2}}{2} \frac{\partial^{3} B_{z 1}}{\partial \xi^{3}}=0
$$

where $B_{z 1}$ is the perturbation of $B_{z}$, and $\xi$ and $\tau$ are stretched coordinates, $\xi=\varepsilon^{1 / 2}\left(x-v_{\mathrm{A}} t\right)$ and $\tau=\varepsilon^{3 / 2} t$ with $\varepsilon \sim\left|B_{z 1} / B_{0}\right|$. This equation has the rarefactive soliton solution,

$$
B_{z 1} / B_{0}=-b_{n} \operatorname{sech}^{2}\left\{\left[x-\left(1-b_{n} / 2\right) v_{\mathrm{A}} t\right] / D_{11}\right\},
$$

where $b_{n}$ is the normalized amplitude, $D_{11}$ is the soliton width,

$$
D_{11}=2 b_{n}^{-1 / 2} d_{11}
$$

Because of Eq. (14), the characteristic wave number of the rarefactive solitary wave can be estimated as

$$
k \sim 1 / D_{11} \sim \varepsilon^{1 / 2} / d_{11}
$$

The dispersion form (10) is valid in the long wavelength region, $k \ll k_{\text {inf }}^{(1)}$. Then, using Eqs. (8) and (15), we obtain a condition for the amplitude of the rarefactive pulse as

$$
\varepsilon \ll \varepsilon_{\max }^{(1-)}
$$

where $\varepsilon_{\max }^{(1-)}$ is defined as

$$
\varepsilon_{\max }^{(1-)}=\frac{3\left(r \cos ^{2} \theta-2 \Delta_{\omega}\right)^{2}}{20 \Delta_{\omega}-2\left(r \cos ^{2} \theta-2 \Delta_{\omega}\right)^{2}} .
$$

The value of $\varepsilon_{\max }^{(1-)}$ increases with decreasing $\theta$ from $\theta_{\mathrm{cl}}$. As $\Delta_{\omega}$ increases, $\varepsilon_{\max }^{(1-)}$ increases. 


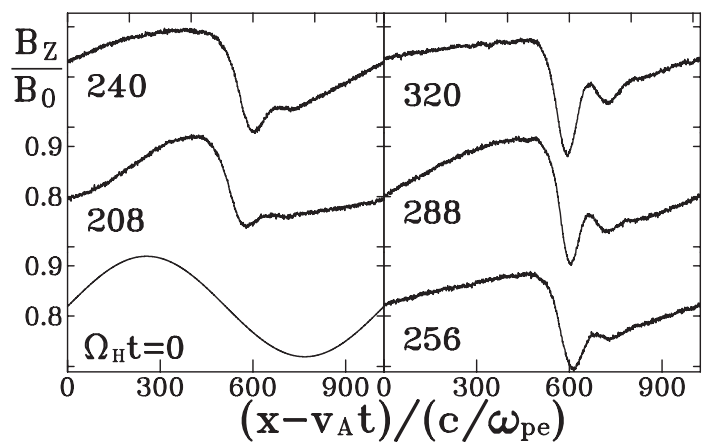

Fig. 2 Wave evolution of the low-frequency mode for $\theta=55^{\circ}$ in H-T plasma with $n_{\mathrm{H}}=n_{\mathrm{T}}$.

\section{Simulation of Nonlinear Evolution}

We study nonlinear evolution of oblique magnetosonic waves with numerical simulations, using a onedimensional (one space coordinate and three velocity components), electromagnetic particle code with full ion and electron dynamics. The system is periodic in the $x$ direction with the length $L_{x}=4096 \Delta_{\mathrm{g}}$, where $\Delta_{\mathrm{g}}$ is the grid spacing. The total number of electrons is $N_{\mathrm{e}} \simeq 2.1 \times 10^{6}$. We simulate $\mathrm{H}-\mathrm{T}$ and $\mathrm{H}-\mathrm{He}$ plasmas. The hydrogen-toelectron mass ratio is taken to be $m_{\mathrm{H}} / m_{\mathrm{e}}=100$. The speed of light is $c /\left(\omega_{\mathrm{pe}} \Delta_{\mathrm{g}}\right)=4$, where $\omega_{\mathrm{pe}}$ is the electron plasma frequency calculated by use of the electron density averaged over the entire region. The electron thermal velocity is $\left(T_{\mathrm{e}} / m_{\mathrm{e}}\right)^{1 / 2} /\left(\omega_{\mathrm{pe}} \Delta_{\mathrm{g}}\right)=0.5$. The ion-to-electron temperature ratio is $T_{\mathrm{i}} / T_{\mathrm{e}}=0.1$. We have chosen this small ratio to avoid ion reflection by nonlinear waves. The external magnetic field is in the $(x, z)$ plane; its strength is $\left|\Omega_{\mathrm{e}}\right| / \omega_{\mathrm{pe}}=1.0$. Initially, we have a sinusoidal disturbance of the low-frequency mode with a wavelength $L_{x}$ propagating in the positive $x$ direction. The amplitude of the magnetic field $B_{z}$ is chosen to be $\delta B_{z} / B_{0}=0.1$; other components of the disturbance are determined according to the linear theory of the low-frequency mode.

We firstly study the rarefactive pulse of the lowfrequency mode in the H-T plasma with $n_{\mathrm{H}} / n_{\mathrm{T}}=1$, taking the propagation angle to be $\theta=55^{\circ}$. The value of $\varepsilon_{\max }^{(1-)}$ for this case is 0.48 ; hence $\delta B_{z} / B_{0}<\varepsilon_{\max }^{(1-)}$ at $t=0$. Figure 2 shows magnetic field profiles at various times. As a result of nonlinear evolution, some pulses are formed. These are the rarefactive pulses of the low-frequency mode. Highfrequency-mode pulses are not found. At $\Omega_{\mathrm{H}} t=320$, the left pulse, whose center is at $\left(x-v_{\mathrm{A}} t\right) /\left(c / \omega_{\mathrm{pe}}\right)=620$, has the amplitude $\varepsilon=\left(B_{\max }-B_{\min }\right) / B_{0}=0.24$, where $B_{\max }$ and $B_{\min }$ are the maximum and minimum values of $B_{z}$. Even though the maximum pulse amplitude $\varepsilon$ is greater than $\delta B_{z} / B_{0}$ of the initial disturbance, it does not exceed $\varepsilon_{\max }^{(1-)}$. Substituting the value of $\varepsilon$ into Eq. (14) yields $D=44 c / \omega_{\text {pe }}$. This is in good agreement with the observed value of the width of the left pulse, $D \simeq 44 c / \omega_{\text {pe }}$. Figure 3 shows the power spectrum $P(k, \omega)$ of magnetic fields calculated from the data for $0<\Omega_{\mathrm{H}} t<320$. The vertical lines

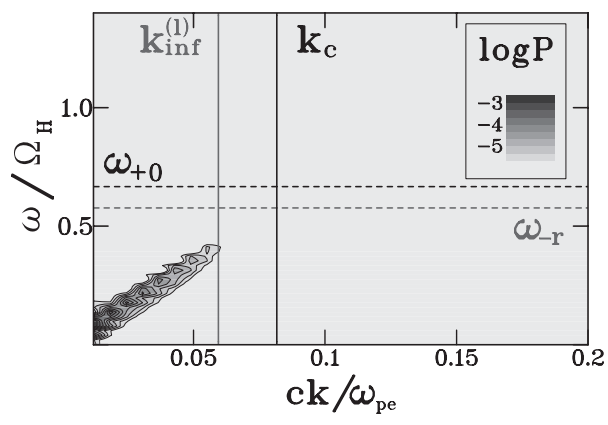

Fig. 3 Power spectrum of magnetic field for $\theta=55^{\circ}$ in H-T plasma with $n_{\mathrm{H}}=n_{\mathrm{T}}$.

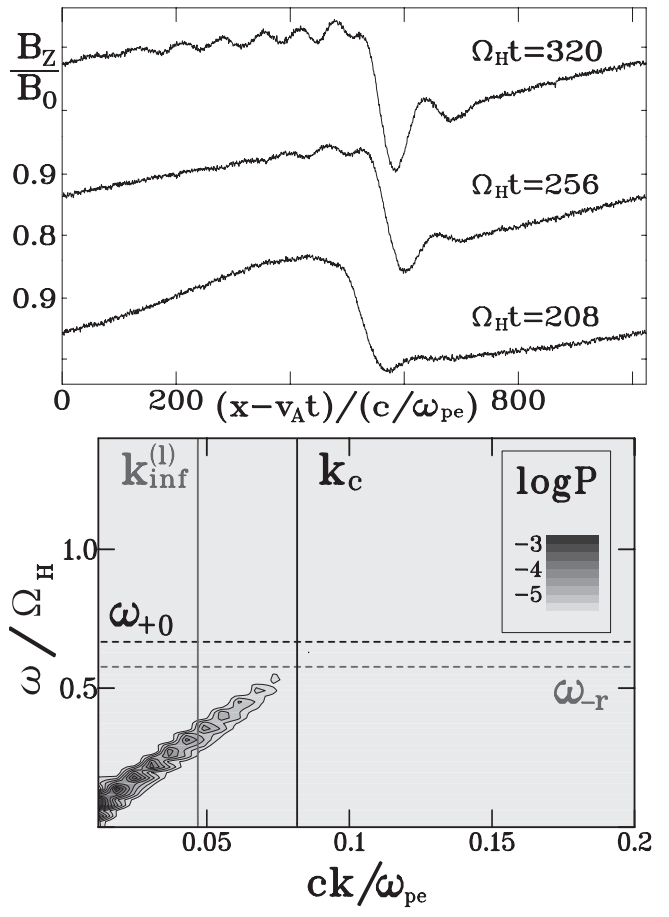

Fig. 4 Wave evolution and power spectrum of magnetic field for $\theta=60^{\circ}$ in H-T plasma with $n_{\mathrm{H}}=n_{\mathrm{T}}$.

represent the wavenumber $k_{\mathrm{c}}$ and $k_{\mathrm{inf}}^{(\mathrm{l})}$, and the horizontal dotted lines represent the frequencies $\omega_{+0}$ and $\omega_{-r}$. This figure verifies that the waves of the low-frequency mode with $k>k_{\text {inf }}^{(\mathrm{l})}$ and of the high-frequency mode with $\omega>\omega_{+0}$ are not generated.

We now consider a case for which the KdV equation (12) is not valid. Figure 4 shows magnetic field profiles at $\Omega_{\mathrm{H}} t=208,256$ and 320 and power spectrum for the case of $\theta=60^{\circ}\left(<\theta_{\mathrm{cl}}\right)$, for which $\varepsilon_{\max }^{(1-)}=0.18$ is greater than the amplitude of the initial sinusoidal wave. As the result of the wave steepening, rarefactive pulses are formed, and the amplitude of the left pulse exceeds $\varepsilon_{\max }^{(1-)}$; for example, at $\Omega_{\mathrm{H}} t=256$, the amplitude is $\varepsilon=\left(B_{\max }-B_{\min }\right) / B_{0}=$ 0.23 . After $\Omega_{\mathrm{H}} t=256$, perturbations are generated behind the left pulse; at $\Omega_{\mathrm{H}} t=320$, the perturbations exist in the region $100<\left(x-v_{\mathrm{A}} t\right) /\left(c / \omega_{\mathrm{pe}}\right)<500$. The power spectrum $P(k, \omega)$ in Fig. 4 shows that the low-frequency-mode waves 


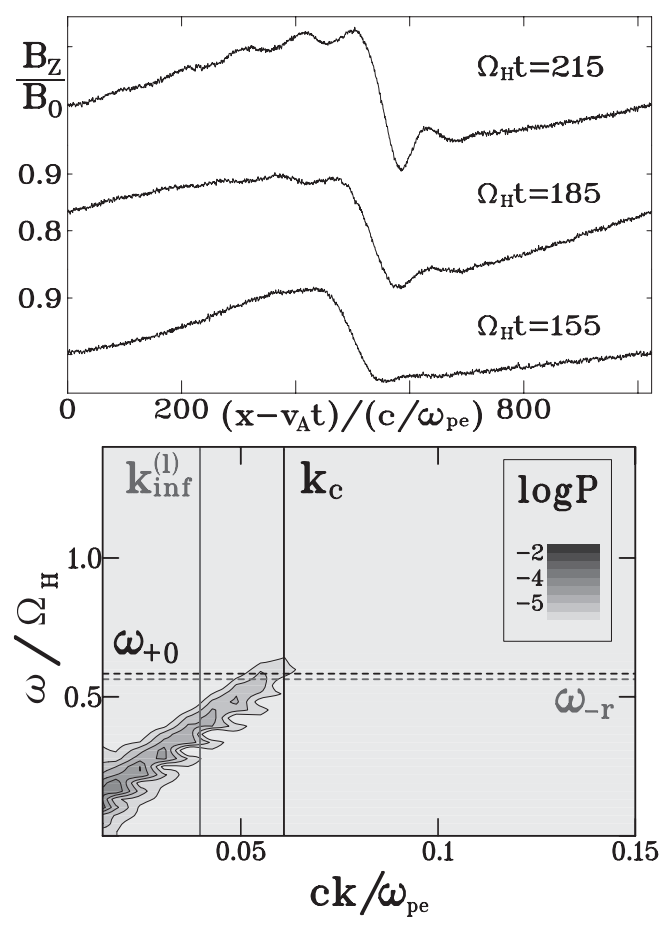

Fig. 5 Wave evolution and power spectrum of magnetic field for $\theta=55^{\circ}$ in $\mathrm{H}-\mathrm{He}$ plasma with $n_{\mathrm{H}}=10 n_{\mathrm{He}}$.

with the wavenumbers $k_{\mathrm{inf}}^{(\mathrm{l})}<k \leq k_{\mathrm{c}}$ are created, in addition to the longer-wavelength waves with $k<k_{\mathrm{inf}}^{(\mathrm{l})}$. This differs from the case with $\theta=55^{\circ}$, in which the waves are limited in the region, $k<k_{\text {inf }}^{(\mathrm{l})}$ (see Fig. 3). We can thus find that when the amplitude of the rarefactive low-frequency-mode pulse exceeds $\varepsilon_{\text {max }}^{(1-)}$, the shorter wavelength $\left(k_{\text {inf }}^{(\mathrm{l})}<k \leq k_{\mathrm{c}}\right)$ perturbations are excited behind the pulse.

We next simulate an H-He plasma with $n_{\mathrm{He}} / n_{\mathrm{H}}=0.1$. Figure 5 shows the result of the case with $\theta=55^{\circ}$. The value of $\varepsilon_{\max }^{(1-)}$ for this case is 0.082 , which is much smaller than those for the cases of the above H-T plasmas. Because of the wave steepening, the maximum amplitude of the low-frequency-mode pulse exceeds $\varepsilon_{\max }^{(1-)}$ (for example, at $\Omega_{\mathrm{H}} t=215$, the amplitude is $\varepsilon=0.23$ ), and the lowfrequency-mode perturbations with $k_{\text {inf }}^{(\mathrm{l})}<k \leq k_{\mathrm{c}}$ are generated behind the rarefactive pulse. Further, power spectrum indicates that the high-frequency-mode waves with $\omega \sim \omega_{+0}$ and $k \sim k_{\mathrm{c}}$ are generated, although their amplitudes are quite small.

The generation of the high-frequency-mode waves is enhanced when $\varepsilon_{\max }^{(1-)}$ is small. We plot in Fig. 6 the result of the case with $\theta=66^{\circ}$, for which $\varepsilon_{\max }^{(1-)}=0.005$. The power spectrum clearly show that the greater-amplitude high-frequency-mode waves with the wavenumbers $k_{\mathrm{c}} \leq k$ are created.

In order to see how the generation of the highfrequency-mode waves depends on $\theta$ in more detail, we compare the results of the cases of the H-He plasma with various $\theta$ 's in the range of $\theta<\theta_{\mathrm{cl}}$. The upper panel in Fig. 7 shows the ratio between the integrals of the powers of the high- and low-frequency modes, $I_{\mathrm{H}} / I_{\mathrm{L}}$, where $I_{\mathrm{H}}$ and $I_{\mathrm{L}}$ are

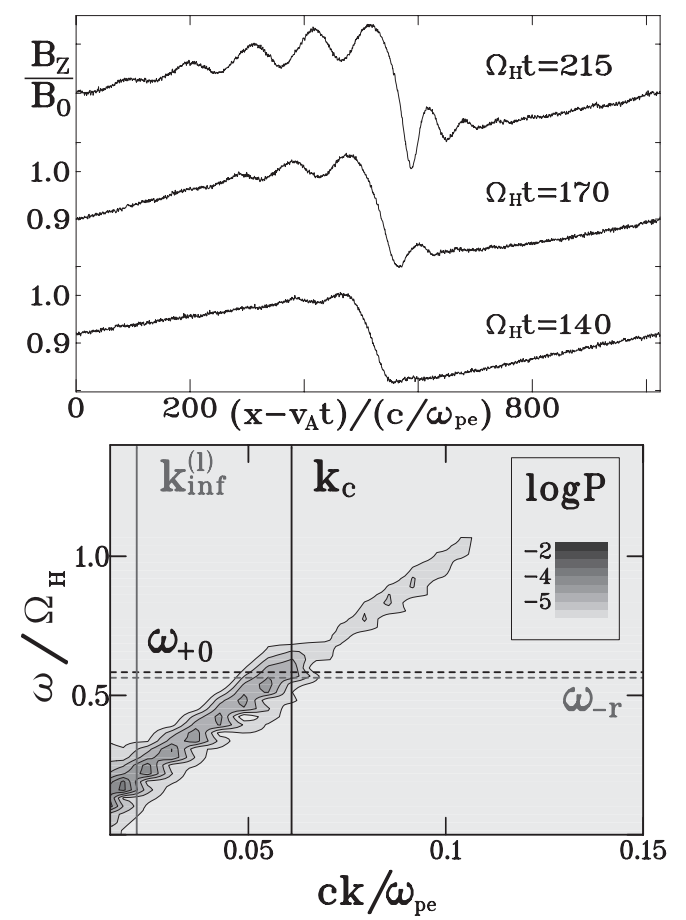

Fig. 6 Wave evolution and power spectrum of magnetic field for $\theta=66^{\circ}$ in $\mathrm{H}-\mathrm{He}$ plasma with $n_{\mathrm{H}}=10 n_{\mathrm{He}}$.

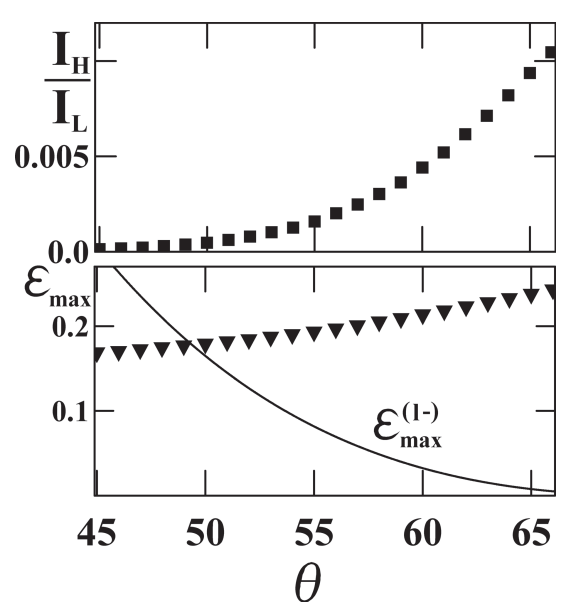

Fig. 7 The ratio $I_{\mathrm{H}} / I_{\mathrm{L}}$ and the maximum amplitude $\varepsilon_{\max }$ for various $\theta$ 's in H-He plasma with $n_{\mathrm{H}}=10 n_{\mathrm{He}}$.

defined by

$$
\begin{aligned}
& I_{\mathrm{H}}=\int_{0}^{k_{\mathrm{m}}} \int_{\omega_{+0}}^{\omega_{+\mathrm{r}}} P(k, \omega) \mathrm{d} k \mathrm{~d} \omega, \\
& I_{\mathrm{L}}=\int_{0}^{k_{\mathrm{m}}} \int_{0}^{\omega_{+0}} P(k, \omega) \mathrm{d} k \mathrm{~d} \omega .
\end{aligned}
$$

Here $\omega_{+\mathrm{r}}$ is the resonance frequency of the high-frequency mode [5], and $k_{\mathrm{m}}=\omega_{+\mathrm{r}} / v_{\mathrm{A}}$. The ratio $I_{\mathrm{H}} / I_{\mathrm{L}}$ is almost zero in the region $\theta<50^{\circ}$, while it increases with $\theta$ in the region $\theta>50^{\circ}$. The lower panel in Fig. 7 shows the maximum amplitude of the magnetic disturbance, $\varepsilon_{\max }=$ $\left(B_{\max }-B_{\min }\right) / B_{0}$; the values of $\varepsilon_{\max }$ are obtained from the data averaged over the period from $\Omega_{\mathrm{H}} t=135$ to 215 . The 
solid line represents $\varepsilon_{\max }^{(1-)}$. For $\theta<50^{\circ}$, the values of $\varepsilon_{\max }$ are smaller than $\varepsilon_{\max }^{(1-)}$, while for $\theta>50^{\circ}, \varepsilon_{\max }$ 's are greater than $\varepsilon_{\max }^{(1-)}$. Comparing upper and lower panels in Fig. 7, we confirm that the production of the high-frequency-mode waves is enhanced as $\varepsilon_{\max }^{(1-)}$ decreases.

\section{Summary}

We have studied the effects of ion composition on oblique magnetosonic waves with theory and simulations. First, we analytically obtained the condition for the $\mathrm{KdV}$ equation for the low-frequency mode to be valid. The upper limit of the amplitudes $\varepsilon_{\max }^{(1-)}$ has been given as a function of $\theta$ and $\Delta_{\omega}$. Next, with electromagnetic particle simulations, we investigated the nonlinear evolution of the waves for various $\theta$ 's and $\Delta_{\omega}$ 's. It was shown that when the amplitude of the long-wavelength rarefactive lowfrequency-mode pulse exceeds $\varepsilon_{\max }^{(1-)}$, shorter-wavelength low- and high-frequency-mode waves are generated.

[1] S.J. Buchsbaum, Phys. Fluids 3, 418 (1960).

[2] M. Toida and Y. Ohsawa, J. Phys. Soc. Jpn. 63, 573, (1994).

[3] M. Toida, Y. Ohsawa and T. Jyounouchi, Phys. Plasmas 2, 3329 (1995).

[4] M. Toida, H. Higashino and Y. Ohsawa, J. Phys. Soc. Jpn. 76, 104502 (2007).

[5] S. Irie and Y. Ohsawa, Phys. Plasmas 10, 1255 (2003). 